\section{Rememorando la contribución de Goldstein y Brown al estudio del metabolismo del colesterol: a un cuarto de siglo del Premio Nobel de Medicina}

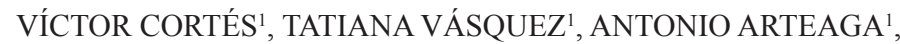 \\ FLAVIO NERVI ${ }^{2}$, ATTILIO RIGOTTI ${ }^{1,2}$
}

\section{The contribution of Goldstein and Brown to the study of cholesterol metabolism}

In December 1985, the Nobel Prize of Medicine was awarded to Drs. Joseph L. Goldstein and Michael S. Brown for their fundamental scientific work on the regulation of cholesterol metabolism mediated by the low density lipoprotein receptor pathway. This article briefly reviews the academic and research accomplishments of Drs. Brown and Goldstein as a tribute to these physician-scientists for their welldeserved award and enormous contribution to biomedical science worldwide.

(Rev Med Chile 2012; 140: 1053-1059).

Key words: Cholesterol; Nobel prize; Receptors, lipoprotein.

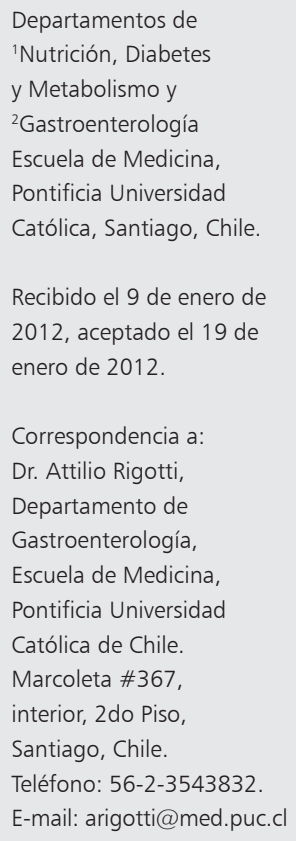

1 l colesterol es una biomolécula que cumple importantes roles metabólicos y fisiológicos en diferentes especies animales, siendo un componente esencial de las membranas celulares y precursor de las hormonas esteroidales y de las sales biliares ${ }^{1}$. Además, el colesterol participa en el control de la morfogénesis embrionaria en los mamíferos ${ }^{2}$. Concordantemente, la homeostasis del colesterol, tanto a nivel celular como del organismo en su totalidad, es un proceso muy relevante y se encuentra finamente regulado. Las alteraciones en la regulación de la síntesis, absorción y excreción del colesterol predisponen al desarrollo de enfermedades crónicas de alta prevalencia, alta mortalidad e impacto social y económico.

La enfermedad cardiovascular isquémica de origen ateroesclerótico es la primera causa de morbilidad y mortalidad en Chile y el mundo. Dentro de su etiopatogenia, la hipercolesterolemia representa un factor de riesgo claramente establecido ya que favorece el desarrollo de ateroesclerosis ${ }^{3}$. Por otra parte, la hipersecreción de colesterol biliar es el evento fisiopatológico clave que determina la aparición de colelitiasis, un estado patológico de alta prevalencia en Chile ${ }^{4}$.

La comprensión detallada de los procesos celulares y moleculares que controlan el metabolismo del colesterol ha sido un logro fundamental para el desarrollo de las estatinas, fármacos hipolipemiantes de amplio uso por su demostrado efecto protector antiaterogénico ${ }^{5}$. En efecto, las estatinas han representado uno de los grandes avances en el armamento terapéutico médico de las últimas décadas, disminuyendo significativamente la mortalidad por enfermedad cardiovascular y cerebrovascular ateroesclerótica ${ }^{3}$.

Dentro de los investigadores más insignes en este campo destacan los médicos Joseph L. Goldstein y Michael S. Brown (Figura 1), cuyo trabajo científico fundacional sobre la regulación del metabolismo del colesterol mediado por la vía celular dependiente del receptor de lipoproteínas de baja densidad (LDLR) los hizo acreedores del Premio Nobel de Medicina en 1985 $5^{6,7}$. Este artículo es un homenaje a estos médicos investigadores con motivo de los 27 años que han transcurrido desde la obtención de este merecido galardón. 
Algunos aspectos históricos sobre la investigación del colesterol previo a 1985

Durante casi dos siglos, la investigación en el campo del colesterol ha mostrado un avance continuo con aportes científicos de alto impacto ${ }^{8}$. En 1815, el químico francés Michel Chevreul propuso el uso del nombre de colesterol (del griego: chole, bilis y stereos, sólido) para designar al compuesto lipídico que había sido detectado por François Poulletier de la Salle en 1769 en los cálculos biliares ${ }^{9}$. Con el transcurso del tiempo y el esfuerzo de muchos investigadores, se estableció la importancia biológica del colesterol y sus derivados hormonales esteroidales y las sales biliares. La definición de la compleja estructura molecular del colesterol y las sales biliares, gracias al trabajo de Heinrich Wieland y Adolf Windaus, y de la intricada vía de biosíntesis del colesterol, estudiada por Konrad Bloch y Feodor Lynen, también les valió el reconocimiento con los Premios Nobel de Química en 1927 y 1928 y el de Medicina en 1964, respectivamente.

En la década de 1930-39, Rudolph Schoenheimer planteó la existencia de un delicado equilibrio entre la cantidad de colesterol que una

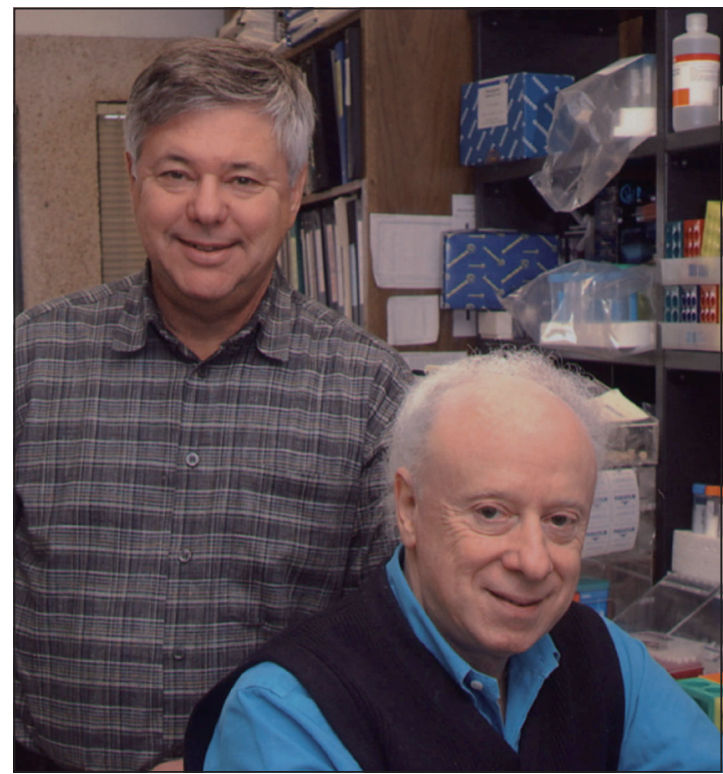

Figura 1. Fotografía de los Dres. Michael Brown (izquierda) y Joseph Goldstein (derecha) tomada en sus laboratorios de investigación del Southwestern Medical Center de la Universidad de Texas en Dallas, USA. célula sintetiza y la que obtiene desde el medio extracelular ${ }^{10}$. Por otro lado, el médico noruego Carl Müller definió en 1938 el cuadro clínico de la hipercolesterolemia familiar como una enfermedad autosómica dominante caracterizada por xantomas, altos niveles de colesterol sanguíneo y enfermedad coronaria precoz ${ }^{11}$. Sin embargo, tanto los mecanismos determinantes de la regulación fisiológica del metabolismo de colesterol así como las causas de la hipercolesterolemia familiar permanecieron desconocidos hasta los años '70 del siglo pasado.

\section{Joseph Goldstein y Michael Brown ${ }^{12-15}$}

Los Drs. Goldstein y Brown se conocieron a mediados de la década de 1960-69 durante su residencia en Medicina Interna en el Massachusetts General Hospital en Boston, forjando una amistad que les ha permitido desarrollar una de las colaboraciones científicas más notables y duraderas del siglo XX y que se extiende por 40 años hasta la actualidad. Posterior a su estadía en Boston, ambos investigadores completaron su formación científica en los National Institutes of Health (NIH) de Estados Unidos de Norteamérica (USA), donde conocieron casos de dislipidemias genéticas, muchos de ellos fatales, que influyeron decisivamente en la elección y desarrollo de su futura línea de investigación. A comienzos de los años 70, el Dr. Goldstein desarrolló un protocolo genético poblacional sobre trastornos lipídicos hereditarios en sobrevivientes de ataques $\operatorname{cardiacos}^{16}$, el cual daría origen al estudio de la hipercolesterolemia familiar. Finalmente, ambos investigadores iniciaron en 1972 una carrera académico-científica conjunta en la Southwestern Medical School de la Universidad de Texas en Dallas, centrados inicialmente en la hipótesis de que las anormalidades en la regulación de la enzima 3-hidroxi-3-metilglutaril CoA (HMGCoA) reductasa, catalizadora de la única etapa limitante de la síntesis del colesterol, era la causa celular de la hipercolesterolemia familiar.

A lo largo de la década de 1970-79 y a pesar de su intenso trabajo científico, los Drs. Brown y Goldstein continuaron desempeñándose como clínicos en los servicios de medicina del Hospital Parkland Memorial de Dallas y haciendo docencia de pre- y postgrado en medicina interna y genética médica. De hecho, la formación de nuevas generaciones de médicos investigadores, así como 
de científicos básicos puros, ha sido unas de sus preocupaciones permanentes, tal como lo manifestaron en una inspiradora publicación de $1997^{17}$. En este trabajo, Brown y Goldstein enfatizan su convicción de la relevancia la participación de médicos clínicos en la investigación de frontera, según ellos la única vía conducente al descubrimiento de los mecanismos celulares y moleculares de enfermedades humanas. Además, este concepto desarrolla un punto central del informe Flexner sobre la formación médica en Estados Unidos de Norteamérica ${ }^{18}$, que recalca la necesidad de la investigación científica ejecutada por profesores de medicina para crear los ambientes académicos propicios e indispensables para una formación médica de calidad. Concordantemente con estas opiniones publicadas hace 15 años, Michael Brown actualmente se desempeña como director del programa combinado de médicos investigadores $(M D-P h D)$ de University of Texas Southwestern Medical Center, participando activamente en la selección y educación de dichos estudiantes y dicta seminarios y congresos especialmente dedicados a la educación biomédica.

\section{Los descubrimientos claves que llevaron al Premio Nobel de Medicina de 1985}

Tanto la biosíntesis celular del colesterol, como la importancia de la enzima HMGCoA reductasa y su regulación por retroalimentación negativa, habían sido previamente delineadas ${ }^{19}$. La aproximación experimental clave aplicada por Brown y Goldstein fue la utilización de cultivo primario de fibroblastos obtenidos desde sujetos controles y pacientes con hipercolesterolemia familiar homocigota $^{20}$. En sus primeras observaciones, Brown y Goldstein notaron que cuando los fibroblastos normales se cultivaban en medios enriquecidos con LDL, la actividad de la enzima HMGCoA reductasa disminuía. Por el contrario, cuando se cultivaban en medios deprivados de LDL, la actividad de HMGCoA reductasa aumentaba, indicando que la actividad de esta enzima dependía de los niveles de exposición de las células al colesterol LDL presente en el medio extracelular. Adicionalmente, ellos establecieron que esta regulación era gatillada específicamente por LDL y no por HDL, sugiriendo la especificidad lipoproteica de este proceso $^{21}$.
En contraste, los fibroblastos de pacientes homocigotos para hipercolesterolemia familiar no mostraron esta retroalimentación: a partir de una actividad enzimática basal de HMGCoA reductasa muy aumentada, la exposición a medios enriquecidos en LDL no suprimía la actividad de esta enzima. Inversamente, la actividad de la reductasa tampoco aumentaba cuando los fibroblastos se cultivaban en ausencia de LDL ${ }^{20}$. Como es lógico, la explicación inicial para estos resultados fue que el defecto observado en las células de los pacientes se debía a una alteración en el gen codificante para la HMGCoA reductasa, lo cual impedía su regulación por colesterol. Sin embargo, la hipótesis correcta surgió después del experimento clave de introducir colesterol directamente en las células, mediante su solubilización en etanol, independientemente de la presencia de partículas de LDL en el medio de cultivo. Con esta intervención, sorprendentemente, se detectó una supresión de la actividad de la reductasa en los fibroblastos de los sujetos con hipercolesterolemia familiar, tal como lo observado en fibroblastos controles normales ${ }^{22}$. Este hallazgo llevó a Goldstein y Brown a postular que el defecto determinante del fenotipo celular asociado a la hipercolesterolemia familiar homocigota residía en una incapacidad de la células para captar colesterol presente en las LDL desde el medio externo hacia el compartimento intracelular, implicando la existencia de un receptor para LDL en la superficie de la membrana plasmática.

La existencia del receptor de LDL (LDLR) se evidenció en 1974 mediante el uso de LDL marcada en su componente proteico con yodo radioactivo $\left({ }^{125} \mathrm{I}\right)^{23}$. Estos experimentos permitieron demostrar la unión saturable y de alta afinidad de LDL a la superficie de células normales en cultivo, la cual se asociaba a internalización y degradación lisosomal de las partículas de LDL, con liberación de colesterol en las células, y reciclaje del receptor hacia la superficie celular (Figura 2). Importantemente, estos fenómenos estaban ausentes en los fibroblastos derivados de pacientes hipercolesterolémicos familiares ${ }^{23}$. Paradójicamente, el trabajo que reportaba estos hallazgos fue inicialmente rechazado para publicación en Journal of Biological Chemistry, una de las más prestigiosas revistas de bioquímica básica, ya que los revisores argumentaron que "la publicación de este artículo con sus observaciones incompletas no servirán a la ciencia médica ni otorgará reconocimiento en el largo plazo 
a sus autores"13, aunque finalmente fue publicado en esta revista en el año $1974^{23}$.

Es interesante destacar que estos descubrimientos clave se lograron pese a los relativamente escasos recursos materiales con que Brown y Goldstein contaban en esa época: un laboratorio con tres mesones, una tecnóloga

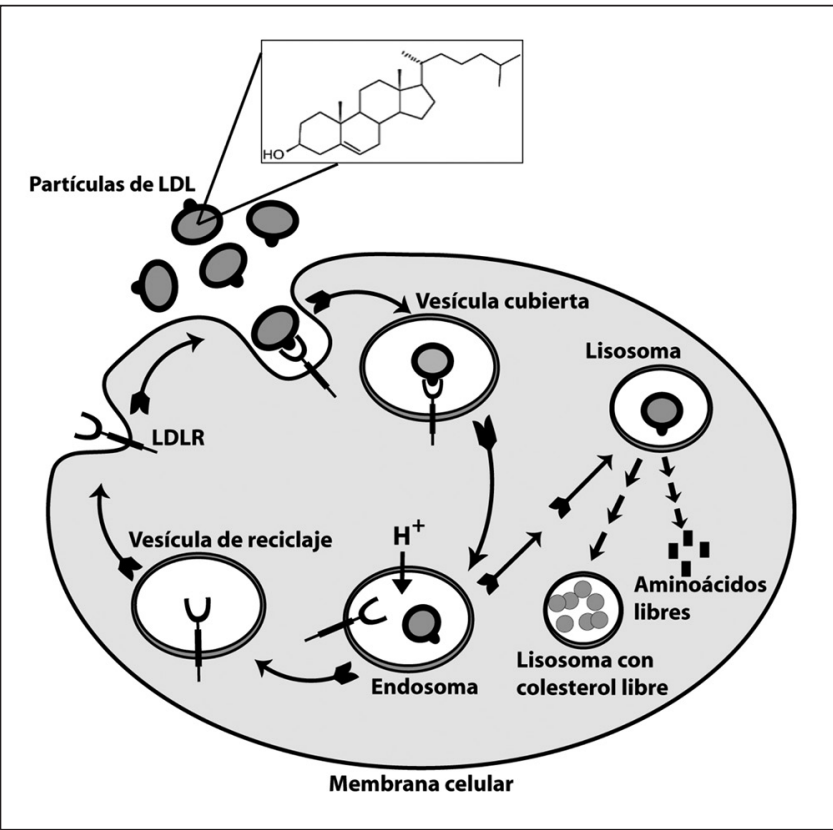

Figura 2. Esquema de endocitosis de LDL, reciclaje del receptor de LDL y el procesamiento intracelular de las partículas de LDL. Las partículas circulantes de LDL, las cuales contienen moléculas de colesterol (recuadro) libre y esterificado, se unen en la superficie celular a los receptores de LDL (LDLR). Estos receptores normalmente se ubican en subdominios de la membrana plasmática conocidos como fosetas cubiertas, por estar asociados en su cara citoplásmica con diversas proteínas intracelulares, de las cuales destaca la clatrina. Una vez producida la asociación entre LDL y su receptor esta foseta es invaginada hacia el espacio intracelular, formándose una vesícula cubierta. Esta estructura se asocia con un nuevo grupo de proteínas intracelulares y sufre acidificación de su espacio luminal, representado aquí por el ingreso de iones $\mathrm{H}^{+}$, formándose un nuevo organelo denominado endosoma. El medio ácido del endosoma permite que las partículas de LDL se disocien del LDLR, el cual es destinado de vuelta a la superficie celular por medio de un organelo denominado vesícula de reciclaje. En la superficie celular, el LDLR es relocalizado en las fosetas cubiertas, quedando disponible para un nuevo ciclo de unión y endocitosis de LDL. A diferencia del LDLR, las partículas de LDL son destinadas hacia los lisosomas para su degradación terminal, en donde el componente lipídico es descompuesto hasta colesterol y ácidos grasos libres para su posterior incorporación en los distintos compartimentos de lípidos metabólicamente activos de la célula, mientras que las apolipoproteínas, en particular la apo B, son hidrolizadas hasta sus aminoácidos constituyentes. Este ciclo de internación y retorno a la superficie celular del LDLR demora aproximadamente 10 min y es repetido cientos de veces en las $20 \mathrm{~h}$ de vida de esta proteína de la membrana plasmática. (Figura modificada en base referencia 7). bioquímica y un auxiliar de laboratorio. Es posible que el éxito de estos trabajos iniciales se produjera por la conjunción afortunada de diversos factores: los talentos personales de Brown y Goldstein, su óptima preparación académica obtenida en ambientes universitarios de calidad, el planteamiento de las preguntas claves, la aplicación de métodos apropiados, la motivación y confianza en sí mismos y una buena dosis de serenditipia.

Junto con una mejor caracterización de la regulación del metabolismo del colesterol, el esfuerzo de Brown y Goldstein y sus colaboradores permitió entender un fenómeno biológico de alcances más generales. En efecto, aunque la existencia de endocitosis celular había sido descrita en décadas previas, este proceso se asumía como inespecífico e independiente de receptores celulares. Por lo tanto, la demostración que la captación celular de LDL requiere una proteína específica en la superficie celular ${ }^{24}$ (Figura 2) constituyó un cambio paradigmático esencial en el estudio de las vías endocíticas de una célula ${ }^{25,26}$.

Adicionalmente, en 1982 Goldstein, Brown y su equipo lograron aislar y purificar el producto proteico del receptor de LDL desde las glándulas suprarrenales de bovino ${ }^{27}$, permitiendo, a posteriori, la identificación del gen codificante para el gen de este receptor en $1985^{28}$, sentando las bases para el análisis de las mutaciones genéticas que dan cuenta de la hipercolesterolemia familiar ${ }^{29}$.

Finalmente, el laboratorio de Brown y Goldstein también identificó por primera vez la existencia de receptores celulares, distintos del LDLR, que median la captación celular de LDL químicamente alteradas, en macrófagos tisulares ${ }^{30}$, un evento patogénico precoz y central en la aterogénesis. Este hallazgo sentó las bases para el estudio de los mecanismos celulares de acumulación anormal de colesterol en la lesión ateromatosa, llevando a la identificación posterior de los receptores scavenger ${ }^{31,32}$ y su papel determinante en la ateroesclerosis ${ }^{33}$. 


\section{La ciencia de excelencia continúa}

En su trabajo posterior al Nobel de Medicina en 1985, Brown y Goldstein centraron sus esfuerzos en el entendimiento de los mecanismos subyacentes a la regulación transcripcional del metabolismo de colesterol (Figura 3). Esto les permitió descubrir el sistema transcripcional conocido como sterol regulatory element binding proteins (SREBPs) ${ }^{34}$. Estos factores transcripcionales interactúan con proteínas reguladoras y responden a cambios en los niveles de colesterol en la membrana del retículo endoplásmico, generando una regulación génica concertada de la síntesis y captación de colesterol LDL mediadas por la enzima HMGCoA reductasa y el receptor de LDL, respectivamente (Figura 3 ).

De manera relevante, la regulación de la expresión del receptor de LDL mediada por SREBP-2 es clave para la acción hipolipemiante de las estatinas. En efecto, las estatinas inhiben competitivamente el sitio activo de la enzima HMGCoA reductasa ${ }^{35}$, disminuyendo la síntesis intracelular de colesterol, lo que conlleva a una tendencia hacia la disminución del contenido de colesterol en la membrana del retículo endoplásmico. Esta reducción es detectada por la maquinaria proteica endoplásmica que activa el sistema transcripcional dependiente de SREBP-2, el cual sufre proteólisis controlada, siendo liberado desde la membrana del retículo endosplásmico y viajando al núcleo celular para estimular compensatoriamente la expresión del gen del receptor de LDL y cantidad del mismo en la superficie celular (Figura 3B). Como consecuencia, el mayor número de receptores de LDL que se localizan en la membrana celular frente a la exposición a las estatinas determina una remoción aumentada de las partículas de LDL y la característica reducción en los niveles plasmáticos de colesterol LDL $\mathrm{L}^{3,5}$. De hecho, el grupo de Brown y Goldstein en colaboración con el Dr. Akira Endo, descubridor de la primera estatina en Japón, fueron quienes

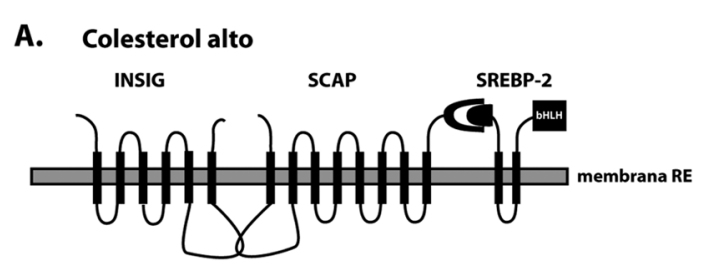

B. Colesterol bajo o en presencia de estatinas

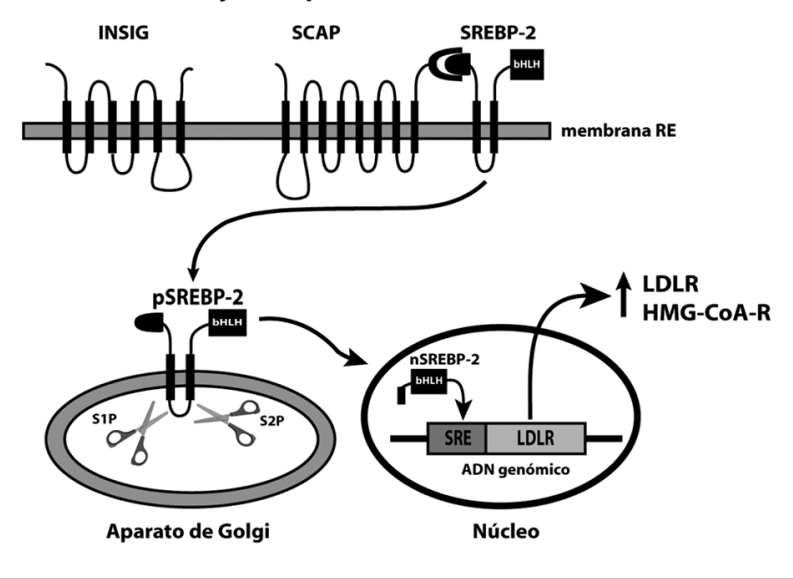

Figura 3. Regulación transcripcional del metabolismo celular del colesterol por el sistema SREBP-2. (A) Cuando el contenido de colesterol celular, principalmente en la membrana del retículo endoplásmico (RE), se encuentra elevado, este lípido se une a la proteína SCAP (SREBP-cleavage activating protein), la cual en respuesta cambia su conformación e interactúa físicamente con INSIG (insulin-induced gene), una proteína residente en el ER. Esta interacción ancla al complejo formado por SCAP y SREBP-2 (sterol regulatory element binding protein-2) en el RE y evita su migración hacia el aparato de Golgi. (B) Cuando el colesterol celular es bajo, SCAP libre de colesterol se desprende de INSIG y el complejo SCAP-SREBP-2 se transloca hacia el Golgi donde SREBP-2 es activado. (C) La activación de SREBP-2 ocurre en el aparato de Golgi y consiste de la liberación del extremo amino terminal de la proteína precursora (pSREBP-2) por acción secuencial de dos proteasas S1P (site 1 protease) y S2P (site 2 protease). Este segmento liberado, el cual contiene el dominio de unión a ADN conocido como bHLH, no está anclado a ninguna membrana celular y se transloca al núcleo celular (nSREBP-2) donde actúa directamente en los elementos de respuesta a esteroles (SRE, sterol responsive elements) que regulan la transcripción del receptor de LDL (LDLR), HMGCOA R (reductasa) y varios otros genes involucrados en la síntesis de colesterol. Esta respuesta transcripcional contribuye a elevar los niveles de colesterol celular y se activa en presencia de estatinas. La mayor expresión del LDLR en la superficie celular inducida por el uso de estatinas trae como consecuencia una reducción de los niveles séricos de colesterol LDL.

establecieron que el efecto farmacológico de este compuesto aislado de un hongo ${ }^{36}$ dependía de esta vía regulatoria de la homeostasis del colesterol celular ${ }^{37}$.

Desde la introducción de las estatinas a la práctica clí- 
nica a comienzos de la década de los años 1980, el efecto hipocolesterolémico de estos medicamentos, que Brown y Goldstein denominan como la "penicilina para el colesterol"38, ha constituido uno de los avances terapéuticos más importantes en la historia de la medicina, así como también una de la fuerzas motoras más poderosas de la industria farmacéutica mundial ${ }^{3}$.

Finalmente, en los últimos años, Brown y Goldstein han concentrado sus estudios en los detalles moleculares de la vía endocítica de captación de colesterol dependiente del receptor de LDL, con espacial énfasis en los mecanismos de acción de las proteínas NPC1 y NPC2, centrales en la solubilización y salida de colesterol desde los lisosomas hacia otros compartimentos intracelulares ${ }^{14,39}$.

\section{Conclusión}

Como se evidencia de este artículo conmemorativo, las casi cuatro décadas de trabajo científico de los Dres. Brown y Goldstein representan fielmente el encanto y la pasión por una idea fija que ha sido abordada desde una perspectiva amplia y sinérgica, alcanzando logros sobresalientes con impacto real para la ciencia, la medicina y la sociedad en general.

Este ilustre ejemplo refleja cabalmente el concepto moderno de ciencia biomédica translacional que fluye recíprocamente tanto desde el gen hacia la enfermedad así como del mesón de laboratorio a la cama del paciente y que los Drs. Brown y Goldstein hicieron realidad en forma pionera. Muchos académicos en diferentes partes del mundo, incluyendo los autores de este artículo, han recibido directa o indirectamente la influencia científica de estos investigadores motivando el desarrollo de una carrera académica centrada en el estudio del metabolismo del colesterol en diferentes enfermedades humanas. Como ellos mismos lo encarnan y lo han expresado, "patientoriented researchers may be bewitched, bothered, and bewildered, but they are still beloved" ${ }^{17}$. ¡Qué sabio y válido consejo para las futuras generaciones de investigadores biomédicos!

Agradecimientos: Los autores agradecen al Dr. Michael Brown por su gentileza de compartir la fotografía reproducida en este artículo.

\section{Referencias}

1. Tabas I. Cholesterol in health and disease. J Clin Invest 2002; 110: 583-90.

2. Willnow TE, Hammes A, Eaton S. Lipoproteins and their receptors in embryonic development: more than cholesterol clearance. Development 2007; 134: 3239-49.

3. Steinberg D. The cholesterol wars: the skeptics vs. the preponderance of evidence. New York, USA: Elsevier Academic Press, 2007.

4. Zanlungo S, Rigotti A, Nervi F. Hepatic cholesterol transport from plasma into bile: implications for gallstone disease. Curr Opin Lipidol 2004; 15: 279-86.

5. Grundy SM. Cholesterol-lowering drugs as cardioprotective agents. Am J Cardiol 1992; 70:27I-32I.

6. The Nobel Prize in Physiology or Medicine 1985. Disponible en: www.nobelprize.org [consultado el 8 de noviembre de 2010].

7. Brown MS, Goldstein JL. A receptor-mediated pathway for cholesterol homeostasis. Science 1986; 232: 34-47.

8. Vance DE, Van den Bosch H. Cholesterol in the year 2000. Biochim Biophys Acta 2000; 29: 1-8.

9. Mayer J, Hanson SD. Michel Eugene Chevreul. J Nutr 1960; 72: 2-7.

10. Schoenheimer R, Breusch F. Synthesis and destruction of cholesterol in the organism. J Biol Chem 1933; 103: 439-48.

11. Müller C. Xanthoma, hypercholesterolemia, angina pectoris. Acta Med Scandinav 1938; 89: 75.

12. Anderson RG. Joe Goldstein and Mike Brown: from cholesterol homeostasis to new paradigms in membrane biology. Trends Cell Biol 2003; 13: 534-9.

13. Kresge N, Simoni RD, Hill RL. 30 years of cholesterol metabolism: the work of Michael Brown and Joseph Goldstein. J Biol Chem 2006; 281: e25-e28.

14. Goldstein JL, Brown MS. The LDL receptor. Arterioscler Thromb Vasc Biol 2009; 29: 431-8.

15. Williams R. Joseph Goldstein and Michael Brown: demoting egos, promoting success. Cir Res 2010; 106: 1006-10.

16. Goldstein JL, Hazzard WR, Schrott HG, Bierman EL, Motulsky AG. Genetics of hyperlipidemia in coronary heart disease. Trans Assoc Am Physicians 1972; 85: 120 38.

17. Goldstein JL, Brown MS. The clinical investigator: bewitched, bothered, and bewildered-but still beloved. J Clin Invest 1997; 99: 2803-12.

18. Mitka M. The Flexner report at the century mark: a wake-up call for reforming medical education. JAMA 2010; 303: 1465-6.

19. Brown MS, Goldstein JL. Cholesterol feedback: from 
Schoenheimer's bottle to Scap's MELADL. J Lipid Res 2009; 50: S15-27.

20. Goldstein JL, Brown MS. Familial hypercholesterolemia: identification of a defect in the regulation of 3-hydroxy3-methylglutaryl coenzyme A reductase activity associated with overproduction of cholesterol. Proc Natl Acad Sci USA 1973; 70: 2804-8.

21. Brown MS, Dana SE, Goldstein JL. Regulation of 3-hydroxy-3- methylglutaryl coenzyme A reductase activity in human fibroblasts by lipoproteins. Proc Natl Acad Sci USA 1973; 70: 2162-6.

22. Brown MS, Dana SE, Goldstein JL. Regulation of 3-hydroxy-3- methylglutaryl coenzyme A reductase activity in cultured human fibroblasts: Comparison of cells from a normal subject and from a patient with homozygous familial hypercholesterolemia. J Biol Chem 1974; 249: 789-96.

23. Goldstein JL, Brown MS. Binding and degradation of low density lipoproteins by cultured human fibroblasts: Comparison of cells from a normal subject and from a patient with homozygous familial hypercholesterolemia. J Biol Chem 1974; 249: 5153-62.

24. Anderson RG, Brown MS, Goldstein JL. Role of the coated endocytic vesicle in the uptake of receptor-bound low density lipoprotein in human fibroblasts. Cell 1977; 10: 351-64.

25. Lodish H, Berk A, Matsudaira P, Kaiser CA, Krieger M, Scott MP, et al. Editores. Molecular Cell Biology, Sexta Edición. New York, USA: WH Freeman and Co., 2007.

26. Alberts B, Johnson A, Lewis J, Raff M, Roberts K, Walter P. Editores. Molecular Biology of the Cell, Quinta Edición. New York, USA: Taylor and Francis Group, 2008.

27. Schneider WJ, Beisiegel U, Goldstein JL, Brown MS. Purification of the low density lipoprotein receptor, an acidic glycoprotein of 164,000 molecular weight. J Biol Chem 1982; 257: 2664-73.

28. Südhof TC, Goldstein JL, Brown MS, Russell DW. The LDL receptor gene: A mosaic of exons shared with different proteins. Science 1985; 228: 815-22.

29. Hobbs HH, Russell DW, Brown MS, Goldstein JL. The
LDL receptor locus in familial hypercholesterolemia: mutational analysis of a membrane protein. Annu Rev Genet 1990; 24: 133-70.

30. Goldstein JL, Ho YK, Basu SK, Brown MS. Binding site on macrophages that mediates uptake and degradation of acetylated low density lipoprotein, producing massive cholesterol deposition. Proc Natl Acad Sci USA 1979; 76: 333-7.

31. Kodama T, Freeman M, Rohrer L, Zabrecky J, Matsudaira P, Krieger M. Type I macrophage scavenger receptor contains alpha-helical and collagen-like coiled coils. Nature 1990; 343: 531-5.

32. Rohrer L, Freeman M, Kodama T, Penman M, Krieger M. Coiled-coil fibrous domains mediate ligand binding by macrophage scavenger receptor type II. Nature 1990; 343: 570-2.

33. Rigotti A. Scavenger receptors and atherosclerosis. Biol Res 2000; 33: 97-103.

34. Goldstein JL, DeBose-Boyd RA, Brown MS. Protein sensors for membrane sterols. Cell 2006; 124: 35-46.

35. Endo A, Kuroda M, Tanzawa K. Competitive inhibition of 3-hydroxy-3-methylglutaryl coenzyme A reductase by ML-236A and ML-236B fungal metabolites, having hypocholesterolemic activity. FEBS Lett 1976; 72: 323-6.

36. Endo A, Kuroda M, Tsujita Y. ML-236A, ML-236B, and ML-236C, new inhibitors of cholesterogenesis produced by Penicillium citrinium. J Antibiot 1976; 29: 1346-8.

37. Brown MS, Faust JR, Goldstein JL, Kaneko I, Endo A. Induction of 3-hydroxy-3-methylglutaryl coenzyme A reductase activity in human fibroblasts incubated with compactin (ML-236B), a competitive inhibitor of the reductase. J Biol Chem 1978; 253: 1121-8.

38. Brown MS, Goldstein JL. A tribute to Akira Endo, discoverer of a "Penicillin" for cholesterol. Atherosclerosis Suppl 2004; 5: 13-6.

39. Infante RE, Wang ML, Radhakrishnan A, Kwon HJ, Brown MS, Goldstein JL. NPC2 facilitates bidirectional transfer of cholesterol between NPC1 and lipid bilayers, a step in cholesterol egress from lysosomes. Proc Natl Acad Sci USA 2008; 105: 15287-92. 